\title{
A new strain rate dependent continuum framework for Mg alloys
}

\author{
Julián Andrés García-Grajales ${ }^{\mathrm{a}, \mathrm{b}}$, Ana Fernández ${ }^{\mathrm{a}, \mathrm{c}}$, Duncan Leary ${ }^{\mathrm{d}}$, Antoine Jérusalem ${ }^{\mathrm{a}, \mathrm{d}}$ \\ ${ }^{a}$ IMDEA Materials Institute, Madrid, Spain \\ ${ }^{b}$ Mathematical Institute, University of Oxford, Oxford, UK \\ ${ }^{c}$ Laboratory for Mechanical Metallurgy, Institute of Materials, Ecole Polytechnique Fédérale de Lausanne, Lausanne, \\ Switzerland \\ ${ }^{d}$ Department of Engineering Science, University of Oxford, Oxford, UK
}

\begin{abstract}
Magnesium (Mg) alloys have recently been put under the spotlight for their specific strength, the highest among structural metals. Although the mechanical behavior of Mg alloys at quasi-static strain rates has been extensively modelled in the recent years, high strain rate studies have been much scarcer. This is mainly due to the rate dependent (RD) mechanical response of their hexagonal close-packed (hcp) crystalline structure. As a result, existing RD models do not account for the differentiated rate sensitivities of each slip and twin systems. Instead, the rate dependency is often conceived as a numerical artefact utilised to smooth the elasto-plastic transition and facilitate the scheme convergence. We thus propose a novel efficient RD crystal plasticity model for hcp metals, applied here to a rolled Mg AZ31 alloy sheet at room temperature. The constitutive RD equations are solved at each time step by either a Newton-Raphson based implicit scheme or an explicit scheme modified so as to ensure convergence independently of the stochastic bursts of slip systems inherent to such approach. Most of the model parameters are taken from the previously calibrated RI model of Fernández et al. (2011), except for the new RD variables (strain rate sensitivity coefficients and reference shear strain rates), taken from the literature when available, or chosen so as to fulfill full compatibility with the the RI model under quasi-static conditions. The model is validated against uniaxial compression tests at high strain rate $\left(10^{3} \mathrm{~s}^{-1}\right)$, in the rolling and normal directions. The results reveal the ability of the model to simulate the mechanical behavior of Mg AZ31 alloy under a very large range of loading rates. To the best of the knowledge of the authors, the proposed model is the first RD continuum model for hcp metals involving physically accurate slip and twin system rate sensitivities, while ensuring convergence.
\end{abstract}

Keywords: Magnesium, continuum modelling, crystal plasticity, strain rate dependency

\section{Introduction}

The amount of efforts to reduce $\mathrm{CO}_{2}$ emissions and their negative environmental impact has increased remarkably during the last decade. In the transport industry more specifically, vehicle weight reduction appears as the most straightforward option to achieve this objective. To this end, magnesium $(\mathrm{Mg})$ and its alloys constitute a significant weight saving material alternative (Mordike and Ebert, 2001; Friedrich and Schumann, 2001; Aghion et al., 2001; Watarai, 2006; Easton et al., 2008; Hirsch and Al-Samman, 2013). However, the use of Mg alloys in transportation industry still requires an exhaustive understanding of their mechanical behavior and microscopic deformation mechanisms under different loading strain rates. Physically based constitutive models should thus be proposed which contemplate the complexity inherent to the deformation of $\mathrm{Mg}$ alloys both at low and high strain rates.

Deformation mechanisms in Mg alloys have been extensively investigated over the past years (Couling et al., 1959; Kocks and Westlake, 1967; Kelley and Hosford, 1968; Chin and Mammel, 1970; Yoo, 1981; Vagaralia and Langdon, 1981; Couret and Caillard, 1985; Yoo and Lee, 1991; Christian and Mahajan, 1995; Agnew et al., 2001; Koike et al., 2003; Barnett et al., 2004; Gehrmann et al., 2005; Agnew and Duygulu, 2005; Keshavarz and Barnett, 2006; Barnett, 2007a,b; del Valle and Ruano, 2007; Lou et al., 2007; Al-Samman and 
Gottstein, 2008; Jain et al., 2008; Hutchinson et al., 2009; Chapuis and Driver, 2011; Fernández et al., 2011; Bettles and Barnett, 2012; Fernández et al., 2013). Their plastic deformation can be accommodated by slips on basal $\{0001\}$, prismatic $\{10 \overline{1} 0\}$, pyramidal $\{10 \overline{1} 1\}\langle a\rangle$, and pyramidal $\{11 \overline{2} 2\}\langle c+a\rangle$ planes. Deformation can also be accommodated by twinning: tensile in $\{10 \overline{1} 2\}$ planes and contraction in $\{10 \overline{1} 1\}$ and $\{10 \overline{1} 3\}$ planes (Christian and Mahajan, 1995; Bettles and Barnett, 2012). The room temperature critical resolved shear stress (CRSS) associated with tensile twinning in pure polycrystalline $\mathrm{Mg}$ and in conventional alloys such as Mg AZ31 alloy (Mg-3wt.\%Al-1wt.\%Zn) is moderately higher than the basal CRSS, but significantly lower than any non-basal (i.e., prismatic, pyramidal $\langle a\rangle$ and pyramidal $\langle c+a\rangle$ ) CRSS. However, compression twins are much less abundant, because the room temperature CRSS corresponding to this deformation mode is relatively high, of the order of the non-basal CRSS (Chapuis and Driver, 2011). They also involve larger shuffles than tensile twinning (Christian and Mahajan, 1995). In agreement with this trend, earlier studies reported that slips on basal planes and $\{10 \overline{1} 2\}$ twinning are the main deformation mechanisms during uniaxial deformation at low temperatures and low strain rates in randomly oriented Mg polycrystals of conventional grain sizes $(\sim 10-50 \mu \mathrm{m})$ (Couling et al., 1959; Kocks and Westlake, 1967; Kelley and Hosford, 1968; Couret and Caillard, 1985; Chin and Mammel, 1970; Yoo, 1981). Non-basal slip systems are also active, albeit to a lesser extent (Koike et al., 2003; Agnew and Duygulu, 2005; Keshavarz and Barnett, 2006). The activity of some of these deformation mechanisms is highly dependent on the temperature and initial texture (Gehrmann et al., 2005; Al-Samman and Gottstein, 2008).

A thorough review of the high strain rate properties of metals and alloys was recently proposed by Armstrong and Walley (2008). The strain rate response of metals is strongly dependent on the crystalline structure (Serra and Bacon, 2005). For body-centred cubic (bcc) metals the strain rate sensitivity coefficient reflects the yield stress dependence, whereas for face-centred cubic (fcc) metals it mainly reflects strain hardening. In the case of hexagonal close-packed metals (hcp), and for the particular case of $\mathrm{Mg}$ alloys with a random texture, it was reported to follow the fcc pattern (Bilby and Crocker, 1965). Building up on these initial observations, extensive experimental campaigns were carried out to characterise more precisely the mechanical behavior of Mg alloys at high strain rates (Mukai et al., 1998; Barnett, 2003; El-Magd and Abouridouane, 2003; Ishikawa et al., 2005a,b; El-Magd and Abouridouane, 2006; Watanabe and Ishikawa, 2009; Tucker et al., 2009; Ulacia et al., 2010; Zhang et al., 2011; Dudamell et al., 2011; Khan et al., 2011; Dudamell et al., 2012). In particular, the mechanical response of Mg AZ31 alloys under dynamic loading condition $\left(10^{3} s^{-1}\right)$ at different temperatures was analysed in detail by Ulacia et al. (2010) and Dudamell et al. (2011). They observed that $\{10 \overline{1} 2\}$ extension twin nucleation and propagation are dramatically enhanced at dynamic rates even at very high temperatures $\left(T>400^{\circ} C\right)$. Conversely, contraction twinning is not favoured at such high rates. This fact can be attributed to the simpler atom shuffles involved in the former mechanism. Furthermore, it was observed that even when grains were not favourably oriented for twinning, \{101ㄹ twinning was taking place at high strain rates, suggesting that the polarity of twinning may be inverted at dynamic rates (Dudamell et al., 2011).

While simulation techniques aimed at describing the mechanical behavior of $\mathrm{Mg}$ alloys at quasi-static strain rates have been extensively proposed (Staroselsky, 1998; Staroselsky and Anand, 2003; Graff et al., 2007; Choi et al., 2007; Tang et al., 2009; Mayama et al., 2009; Choi et al., 2009, 2010b,a; Lévesque et al., 2010; Fernández et al., 2011, 2013; Herrera-Solaza et al., 2014; Juan et al., 2014), the use of these models at dynamic strain rates has still not been thoroughly explored. Among several non-crystal plasticity phenomenological constitutive models (Johnson and Cook, 1985; Zerilli and Armstrong, 1987; El-Magd and Abouridouane, 2006; Li, 2012; Gao et al., 2014), the ones proposed by Johnson and Cook (1985) and Zerilli and Armstrong (1987) have been often used due to their simplicity and inexpensive computational cost. However, by nature, microstructural evolution studies, such as texture evolution, slip/twin activities or intragranular mechanical fields are not accessible to these models. To this end, physically based constitutive models such as crystal plasticity continuum models have been used to enable the investigation of a variety of aspects of the heterogeneous plastic response of polycrystals, including the complex interactions between grains and their influence on the macroscopic texture. Along these lines, fcc and bcc rate independent (RI) crystal plasticity continuum models have been proposed by many research groups (Cuitiño and Ortiz, 1992; Anand and Kothari, 1996; Anand, 2004; Houtte et al., 2006; Zhao et al., 2007; Kuchnicki et al., 2008; Jérusalem et al., 2008; Roters et al., 2010; Lee et al., 2010; Liu et al., 2011; Thamburaja, 2010; Rossiter et al., 2010; 
Zamiri and Pourboghrat, 2010; Watanabe et al., 2010; Sung et al., 2010), with similar efforts for hcp metals (Staroselsky, 1998; Staroselsky and Anand, 2003; Graff et al., 2007; Mayeur and McDowell, 2007; Choi et al., 2007; Tang et al., 2009; Mayama et al., 2009; Prakasha et al., 2009; Choi et al., 2009, 2010b,a; Lévesque et al., 2010; Fernández et al., 2011; Choi et al., 2011; Beyerlein et al., 2011; Izadbakhsh et al., 2011; Abdolvand and Daymond, 2013a,b; Fernández et al., 2013; Herrera-Solaza et al., 2014). However, by definition, RI models cannot account for rate dependent (RD) effects and thus cannot a priori be used for dynamic problems. To this end, RD formulations linking directly each slip system's resolved shear stress (RSS) to its instantaneous slip rate have also been proposed (Hutchinson, 1976; Peirce et al., 1982, 1983; Asaro and Needleman, 1985; Nemat-Nasser and Obata, 1986). The integration of a RD crystal plasticity model then requires iterative solution procedures on all slip systems at each time step often resulting in severe numerical instabilities in the resolution of the constitutive equations, especially when different rate sensitivities are used for the different systems. Cuitiño and Ortiz (1992) introduced a Newton-Raphson method where a line-search procedure was used to guarantee numerical stability. Subsequent improvements of the latter formulation were carried out by several authors (Ling et al., 2005; Kuchnicki et al., 2006; Zhao et al., 2007). McGinty and McDowell (2006) proposed a semi-implicit integration scheme for the identification of the active slip systems prior to determining the shear rates, which facilitates the subsequent determination of the current slip rates. Yoon et al. (2007) used an implicit deformation gradient approach to update the stresses and texture orientations. Li et al. (2008) proposed an homotopy continuation method combined with a NewtonRaphson iteration procedure to improve the numerical robustness. However, despite the recent interest in the mechanical response of $\mathrm{Mg}$ alloys under high strain rates, the previously mentioned continuum models were only applied to cubic metals. The consideration of hep metals involves the additional complexity of having different rate sensitivities between slip and twin systems. Numerically, this effectively leads to additional numerical instabilities. To avoid this, Pandey et al. (2015) and Kabirian et al. (2015) used a visco-plastic self-consistent polycrystal model (Choi et al. $(2007,2009)$ ) to simulate the mechanical responses and the evolution of the initial texture in a Mg AZ31 alloy sheet during for various loading conditions by adopting different hardening coefficients to fit the macroscopic flow curve and texture evolution for different strain rates. To the best of the knowledge of the authors, there is thus no crystal plasticity model able to reproduce the mechanical behavior at all strain rates of hcp metals without recalibration of material parameters. Said otherwise, a universal crystal plasticity model adequate at dynamic rates and using the same material parameters as in quasi-static conditions is still missing.

A RI crystal plasticity finite element (CPFE) model aimed at fully describing the activity of slip and twinning systems, while accounting for their interactions through hardening mechanisms, was developed and implemented for Mg AZ31 alloy by Fernández et al. (2011). This model was calibrated and validated against a set of quasi-static compression tests at room temperature along the rolling and normal directions. It was then exploited by investigating stress and strain fields, texture evolution, and slip and twin activities during deformation. This model was later extended to represent a more realistic polycrystal by use of 3D Voronoï tessellations with a large number of elements, allowing for the high resolution study of the local intragranular mechanical fields (Fernández et al., 2013). In particular, it was utilised to investigate the effect of grain boundary misorientation on tensile twin propagation in a rolled Mg AZ31 alloy sheet. In this work, the same model is adopted and modified to account for RD effects. Two numerical integration schemes are proposed to solve the resultant constitutive model equations: explicit (incremental formulation) and implicit (Newton-Raphson method). The parameters of the RD model are taken as the same as their calibrated RI counterparts (Fernández et al., 2011) when common, whereas the values for the strain rate sensitivity coefficients and the reference strain rates are taken from the literature and determined so as to ensure compatibility between the RI and RD formulations, respectively. The model is ultimately validated against uniaxial compression tests at high strain rate $\left(10^{3} \mathrm{~s}^{-1}\right)$ performed for a textured sample of Mg AZ31 alloy along both rolling and normal directions (Ulacia et al., 2010).

The outline of the paper is as follows. The formulation of the proposed RD constitutive model is summarised in Section 2. Section 3 introduces the two different numerical approaches (explicit and implicit) proposed to solve the resultant equations. The results of the numerical simulations are presented in Section 4, and their discussion is given in Section 5. Finally, some concluding remarks are collected in Section 6 . 


\section{Constitutive model}

The constitutive framework used in this work is based on the RI crystal plasticity model originally developed by Staroselsky and Anand (2003) (see also Staroselsky (1998)). Fernández et al. (2011) improved this model by adding differentiated self- and cross-hardening between slip and twin systems and focussed on the individual calibration of each one of the interaction parameters. The proposed evolution into a RD model implies drastic differences in the resultant equations, and thus new resolution methods have to be implemented. In the following, we provide a summary of the constitutive formulation, highlighting the relevant concepts related to the RD specificities.

\subsection{Constitutive relations}

The deformation gradient tensor $\mathbf{F}$ at time $t$ can be decomposed multiplicatively into an elastic contribution $\mathbf{F}^{e}$, related to the elastic distortion of the lattice, and a plastic contribution $\mathbf{F}^{p}$, representing the cumulative effects of the non-reversible deformation in the crystal (Lee, 1969):

$$
\mathbf{F}(t)=\mathbf{F}^{e}(t) \cdot \mathbf{F}^{p}(t)
$$

where $\operatorname{det}\left(\mathbf{F}^{e}\right)>0$ and $\operatorname{det}\left(\mathbf{F}^{p}\right)=1$ (Staroselsky and Anand, 2003).

The stress is described by a second order Piola-Kirchoff tensor $\mathbf{S}^{e}$ defined in the intermediary referential (Malvern, 1969) which, in metals, follows a linear relationship:

$$
\mathbf{S}^{e}=\mathcal{C}: \mathbf{E}^{e}
$$

where $\mathcal{C}$ is a fourth order anisotropic elasticity tensor, and $\mathbf{E}^{e}$ is the elastic Green-Lagrange deformation tensor:

$$
\mathbf{E}^{e}=\frac{1}{2}\left(\mathbf{C}^{e}-\mathbf{I}\right)
$$

where $\mathbf{C}^{e}$ is the right Cauchy-Green tensor defined as:

$$
\mathbf{C}^{e}=\mathbf{F}^{e T} \cdot \mathbf{F}^{e}
$$

Finally, the plastic velocity gradient tensor $\mathbf{L}^{p}=\dot{\mathbf{F}}^{p} \cdot \mathbf{F}^{p-1}$ is defined as:

$$
\mathbf{L}^{p}=\sum_{i} \operatorname{sign}\left(\tau_{i}\right) \dot{\gamma}_{i} \mathbf{S}_{0}^{i}+\sum_{\alpha} \operatorname{sign}\left(\tau_{\alpha}\right) \dot{\gamma}_{\alpha} \mathbf{S}_{0}^{\alpha}
$$

where $\dot{\gamma}_{i}$ and $\dot{\gamma}_{\alpha}$ are the shear strain rates and $\mathbf{S}_{0}^{i}$ and $\mathbf{S}_{0}^{\alpha}$ are the Schmid tensors for slip system $i$ and twin system $\alpha$ in the reference configuration:

$$
\left\{\begin{array}{l}
\mathbf{S}_{0}^{i}=\mathbf{m}_{0}^{i} \otimes \mathbf{n}_{0}^{i} \\
\mathbf{S}_{0}^{\alpha}=\mathbf{m}_{0}^{\alpha} \otimes \mathbf{n}_{0}^{\alpha}
\end{array}\right.
$$

where $\mathbf{n}_{0}^{i / \alpha}$ and $\mathbf{m}_{0}^{i / \alpha}$ are the unit normal to the slip/twin plane, and unit vector of the slip/twin direction in the reference configuration, respectively. See Figure 1 for a schematic of the corresponding systems.

\subsection{Incremental problem}

The plastic deformation gradient tensor at time $\tau=t+\Delta t$, where $\Delta t$ is the time step, can be given from its counterpart at time $t, \mathbf{F}^{p}(t)$, by (Staroselsky, 1998):

$$
\mathbf{F}^{p}(\tau)=\mathbf{F}_{\Delta t}^{p} \cdot \mathbf{F}^{p}(t)
$$

where $\mathbf{F}_{\Delta t}^{p}$ is given by the kinematic incremental relation (Staroselsky, 1998):

$$
\mathbf{F}_{\Delta t}^{p}=1+\sum_{i} \Delta \gamma_{i} \mathbf{S}_{0}^{i}+\sum_{\alpha} \Delta \gamma_{\alpha} \mathbf{S}_{0}^{\alpha}
$$




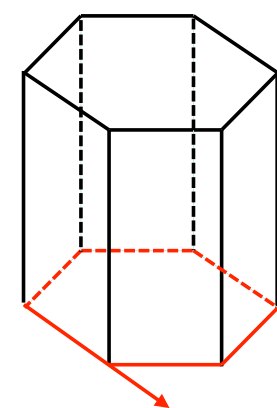

Basal $\langle a\rangle$

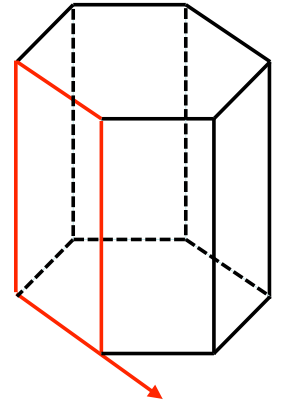

Prismatic $\langle a\rangle$

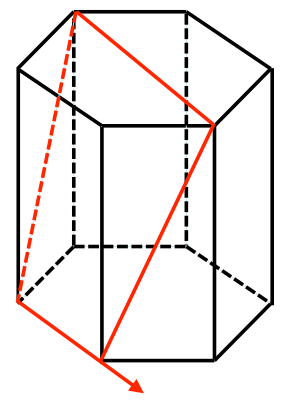

Pyramidal $\langle a\rangle$

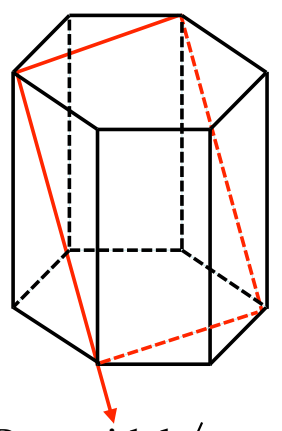

Pyramidal $\langle c+a\rangle$

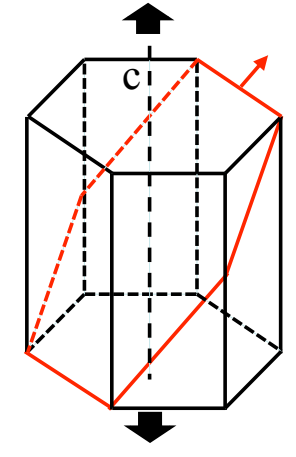

Tensile twin

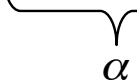

Figure 1: Schematic of the four hcp slip systems considered here $(i)$, along with the tensile twinning systems $(\alpha)$.

where $\Delta \gamma_{i}$ and $\Delta \gamma_{\alpha}$ are the shear strain increments.

The RSSs for all slip systems $i$ and twin systems $\alpha$ are defined as (Staroselsky, 1998):

$$
\left\{\begin{aligned}
\tau_{i} & =\left(\mathbf{C}^{e}: \mathbf{S}^{e}\right) \cdot \mathbf{S}_{0}^{i} \\
\tau_{\alpha} & =\left(\mathbf{C}^{e}: \mathbf{S}^{e}\right) \cdot \mathbf{S}_{0}^{\alpha}
\end{aligned}\right.
$$

At time $t$ all variables of the problem $\left(\mathbf{F}(t), \mathbf{F}^{p}(t), \mathbf{S}^{e}(t), \mathbf{S}_{0}^{i}(t)\right.$ and $\left.\mathbf{S}_{0}^{\alpha}(t)\right)$ are known, whereas at time $\tau$ only $\mathbf{F}(\tau)$ is provided. Therefore, the incremental problem consists in calculating the rest of the variables, see Staroselsky (1998); Staroselsky and Anand (2003); Fernández et al. (2011) for more details. In order to undertake such calculation, a set of "trial" quantities are defined as:

$$
\left\{\begin{array}{l}
\mathbf{F}^{e}(\tau)^{t r}=\mathbf{F}(\tau) \cdot \mathbf{F}^{p}(t)^{-1} \\
\mathbf{C}^{e}(\tau)^{t r}=\mathbf{F}^{e T}(\tau)^{t r} \cdot \mathbf{F}^{e}(\tau)^{t r} \\
\mathbf{E}^{e}(\tau)^{t r}=\frac{1}{2}\left(\mathbf{C}^{e}(\tau)^{t r}-\mathbf{I}\right) \\
\mathbf{S}^{e}(\tau)^{t r}=\mathcal{C}: \mathbf{E}^{e}(\tau)^{t r}
\end{array}\right.
$$

The trial RSSs at time $\tau$ for all slip systems $i$ and twin systems $\alpha$ are then defined as:

$$
\left\{\begin{aligned}
\tau_{i}(\tau)^{t r} & =\mathbf{S}^{e}(\tau)^{t r} \cdot \mathbf{S}_{0}^{i}(t) \\
\tau_{\alpha}(\tau)^{t r} & =\mathbf{S}^{e}(\tau)^{t r} \cdot \mathbf{S}_{0}^{\alpha}(t)
\end{aligned}\right.
$$

Gathering Equations (7), (10) and (11), and assuming that $\operatorname{sign}\left(\tau_{i}(\tau)^{\operatorname{tr}}\right)=\operatorname{sign}\left(\tau_{i}(\tau)\right)$ and $\operatorname{sign}\left(\tau_{\alpha}(\tau)^{\operatorname{tr}}\right)=$ $\operatorname{sign}\left(\tau_{\alpha}(\tau)\right)$ for slip and twin systems, respectively, the following constitutive relation is obtained:

$$
\left|\tau_{i}(\tau)^{t r}\right|=\left|\tau_{i}(\tau)\right|+\sum_{j \in \mathcal{N}}\left\{\operatorname{sign}\left(\tau_{i}(\tau)^{t r}\right) \operatorname{sign}\left(\tau_{j}(\tau)^{t r}\right) \mathbf{S}_{0}^{i}(t): \mathcal{C}:\left[\operatorname{sym}\left(\mathbf{C}^{e}(\tau)^{t r} \cdot \mathbf{S}_{0}^{j}(t)\right)\right]\right\} \Delta \gamma_{j}
$$

where $\mathcal{N}$ is the set of activated systems in the crystal. For clarity, here and subsequently, both slip and twin systems are indiscriminately referred to as $i$ or $j$. 


\subsection{Strain rate dependency law}

In RD formulations (Hutchinson, 1976; Asaro and Needleman, 1985; Cuitiño and Ortiz, 1992; Zhao et al., 2007), the consistency condition is accomplished by establishing a relation between slip/twin rates and the corresponding instantaneous RSSs. In this work, the shear rate deformation on slip/twin systems is given by the following power law flow rule (Zhao et al., 2007):

$$
\dot{\gamma}_{i}= \begin{cases}\dot{\gamma}_{i}^{0}\left[\left(\frac{\tau_{i}}{s_{i}}\right)^{\frac{1}{m_{i}}}-1\right] & \text { if } \tau_{i} \geq s_{i} \\ 0 & \text { otherwise }\end{cases}
$$

where for each system $i, s_{i}$ is the CRSS, $\dot{\gamma}_{i}^{0}$ the reference shear rate and $m_{i}$ the strain rate sensitivity coefficient. As a consequence, a system $i$ is considered activated when $\dot{\gamma}_{i}>0$, i.e., when $\Delta \gamma_{i}>0$. As noted by several authors (Ling et al., 2005; McGinty and McDowell, 2006), the previous equation is slightly different from the one proposed by Cuitiño and Ortiz (1992). In this equation, the initial yielding is shifted to mitigate the tendency of the original model proposed by Hutchinson (1976) to predict unrealistic values of shear rate. Additionally, this shifted relation is also numerically more stable.

Along with the incremental hardening relationship,

$$
s_{i}(\tau)=s_{i}(t)+\underbrace{\sum_{j \in \mathcal{N}} h_{i j}(t) \Delta \gamma_{j}}_{\Delta s_{i}}
$$

where $h_{i j}(t)$ is the hardening matrix at time $t$, Equation (12) can then be rewritten as:

$$
\begin{aligned}
\sum_{j \in \mathcal{N}}\left\{\operatorname{sign}\left(\tau_{i}(\tau)^{t r}\right) \operatorname{sign}\left(\tau_{j}(\tau)^{t r}\right) \mathbf{S}_{0}^{i}(t): \mathcal{C}:\left[\operatorname{sym}\left(\mathbf{C}^{e}(\tau)^{t r} \cdot \mathbf{S}_{0}^{j}(t)\right)\right]+\right. & \left.h_{i j}(t)\left(\frac{\Delta \gamma_{i}}{\Delta t \dot{\gamma}_{i}^{0}}+1\right)^{m_{i}}\right\} \Delta \gamma_{j} \\
& =\left|\tau_{i}(\tau)^{t r}\right|-s_{i}(t)\left(\frac{\Delta \gamma_{i}}{\Delta t \dot{\gamma}_{i}^{0}}+1\right)^{m_{i}}
\end{aligned}
$$

Note that, as opposed to the RI formulation (Fernández et al., 2011), where the consistency condition leads to a system of linear equations at each time step, this system is nonlinear.

\section{Implementation}

Equation (15) is an ill-conditioned system of nonlinear equations whose resolution is not straightforward. Several issues can be identified. For a typical time step, strain increment values vary within a wide range, from $10^{-6}$ for fully activated mechanisms to $10^{-15}$ (or machine precision) for the mechanisms which are not favourably activated. The system of equations is a priori lacking homogeneity because of the potential different orders of magnitude between the different $m_{i}$ of each deformation mechanism, e.g., for basal slip and twin systems they are almost zero (Watanabe and Ishikawa, 2009), while for non-basal slip systems they are of the order of $10^{-2}$.

For these reasons, two different approaches are proposed to tackle the resolution of Equation (15): explicit and implicit. In the former scheme, the shear rate is calculated explicitly and thus the system becomes a system of linear equations that can easily be solved (with the same techniques as in the RI constitutive model), whereas in the latter, the Newton-Raphson method is implemented to directly solve the system.

\subsection{Explicit scheme}

This approach was initially motivated by the complexity of the system of equations, and the Finite Element Method (FEM) explicit time integration solver used here, which already requires a small time 
step $^{1}$. This method a priori relies on the explicit calculation of $\dot{\gamma}_{i}=\frac{\Delta \gamma_{i}}{\Delta t}$ at each time step and each system by using the strain rate $\dot{\gamma}_{i}$ of the previous time step in Equation (15). Doing so, the equation becomes a linear system of equations in $\Delta \gamma_{j}$. A problem arises when $\dot{\gamma}_{i}=0$, i.e., $\Delta \gamma_{i}=0$. Indeed, if the system remains consistently inactivated for physical reasons for many steps, its future activation (where $\Delta \gamma_{j}>0$ in Equation (15)) will incorrectly includes its past inactivation by setting $\dot{\gamma}_{i}=0$. Additionally, if it just stopped being activated for a few steps for stochastic reasons, its reactivation will wrongly assume that it has not been activated in the near past. A possible solution to this problem consists in holding $\dot{\gamma}_{i}$ constant until a new non-zero $\Delta \gamma_{i}$ is produced. However, this technique will reversibly affect cases where non-activation is physically justified during a given period of time (e.g., cyclic loading). A second solution consists in redistributing the last non-zero $\Delta \gamma_{i}$ on the number of time steps that $\Delta \gamma_{i}$ remains zero, effectively "lengthening" the activation by diluting it in the non-activation periods. The problem with this approach is related to the RD activation of the deformation mechanisms: after a long period of inactivation, sudden long activations are underestimated, and after a short period of inactivation, small activations are overestimated.

To this end, a variable moving average algorithm is proposed to undertake the averaging process based on a predetermined shear window. This shear window $\tilde{\gamma}$ is representative of the maximum increment of shear for which a given shear rate will have a rate dependent influence. This approach naturally implies a longer averaging time window for slow rates and a smaller one for fast rates. At each time step:

1. The maximum shear rate $\dot{\gamma}_{\text {max }}$ is defined as:

$$
\dot{\gamma}_{\max }=\max _{i \in \mathcal{N}}\left(\dot{\gamma}_{i}\right)
$$

2. The corresponding instantaneous time window $T_{\max }$ corresponding to the shear window $\tilde{\gamma}$ can then be evaluated from:

$$
T_{\max }=\frac{\tilde{\gamma}}{\dot{\gamma}_{\max }}
$$

3. The size of the window in number of time steps is recalculated at the next iteration as a function of $T_{\text {max }}$ by means of a counter $n_{i}$ for each system $i$ :

$$
n_{i}= \begin{cases}n_{i}+1 & \text { if }\left(n_{i}+1\right) \Delta t<T_{\max } \\ \frac{T_{\max }}{\Delta t} & \text { otherwise }\end{cases}
$$

This amounts to ramping up the averaging period at the beginning of the simulation until it reaches $T_{\max }$ and correcting it when needed later on. Note that $\Delta t$ is assumed to remain constant during $T_{\text {max }}$, and that $n_{i}$ thus doesn't necessarily remain an integer (which doesn't affect the algorithm).

4. The shear rate of the corresponding system $i$ is calculated at the end of the time step as:

$$
\dot{\gamma}_{i}=\frac{\dot{\gamma}_{i}^{\text {old }}\left(n_{i}-1\right)+\frac{\Delta \gamma_{i}}{\Delta t}}{n_{i}}
$$

where $\dot{\gamma}_{i}^{\text {old }}$ is the shear rate at the previous step.

5. The current value is stored for the next time step:

$$
\dot{\gamma}_{i}^{\text {old }}=\dot{\gamma}_{i}
$$

Recalling $\mathbf{A}$ and $\mathbf{b}$ as the matrix and right hand side corresponding to the RI formulation (Fernández et al., 2011),

$$
\left\{\begin{aligned}
A_{i j} & =\operatorname{sign}\left(\tau_{i}(\tau)^{\operatorname{tr}}\right) \operatorname{sign}\left(\tau_{j}(\tau)^{t r}\right) \mathbf{S}_{0}^{i}(t): \mathcal{C}:\left[\operatorname{sym}\left(\mathbf{C}^{e}(\tau)^{t r} \cdot \mathbf{S}_{0}^{j}(t)\right)\right]+h_{i j} \\
b_{i} & =\left|\tau_{i}(\tau)^{t r}\right|-s_{i}(t)
\end{aligned}\right.
$$

\footnotetext{
${ }^{1}$ It should be emphasised that the proposed explicit and implicit approaches are for the constitutive Equation (15); the FEM solver remains explicit in time.
} 
the RI problem consists in solving for the vector of slip/twin increments $\boldsymbol{\Delta} \gamma$ in:

$$
\left\{\begin{aligned}
\boldsymbol{A} \cdot \Delta \boldsymbol{\gamma} & =\boldsymbol{b} \\
\Delta \gamma_{j} & \geq 0, \forall j \in \mathcal{N}
\end{aligned}\right.
$$

Their counterparts can then be defined for the RD case from Equation (15) as:

$$
\left\{\begin{array}{c}
\mathcal{A}_{i j}=A_{i j}+h_{i j}\left(\left(\frac{\Delta \gamma_{i}}{\Delta t \dot{\gamma}_{i}^{0}}+1\right)^{m_{i}}-1\right) \\
\mathfrak{b}_{i}=b_{i}+s_{i}(t)\left(1-\left(\frac{\Delta \gamma_{i}}{\Delta t \dot{\gamma}_{i}^{0}}+1\right)^{m_{i}}\right)
\end{array}\right.
$$

where $\frac{\Delta \gamma_{i}}{\Delta t}$ is replaced by the shear rate $\dot{\gamma}_{i}$ defined explicitly by the algorithm presented above. The system of linear equations

$$
\left\{\begin{aligned}
\mathcal{A} \cdot \Delta \gamma & =\mathfrak{b} \\
\Delta \gamma_{j} & \geq 0, \forall j \in \mathcal{N}
\end{aligned}\right.
$$

is finally solved numerically using the singular value decomposition (SVD) method (Press, 1992).

\subsection{Implicit resolution}

Equation (15) is an ill-conditioned system of nonlinear equations in which all values depend on the unknown variables $\Delta \gamma_{i}$. Without any explicit approximation, Equation (24) is a system composed by $\mathcal{A}(\Delta \gamma), \mathfrak{b}(\Delta \gamma)$ and $\Delta \gamma$ as:

$$
\left\{\begin{aligned}
\mathcal{A}(\Delta \gamma) \cdot \Delta \gamma & =\mathfrak{b}(\Delta \gamma) \\
\Delta \gamma_{j} & \geq 0, \forall j \in \mathcal{N}
\end{aligned}\right.
$$

Equation (25) is numerically solved by use of the Newton-Raphson method with line search and backtracking strategies (Press, 1992). To do so, the function to minimise is defined as follows:

$$
f_{i}\left(\Delta \gamma_{j}\right)=\mathcal{A}_{i j}\left(\Delta \gamma_{j}\right) \Delta \gamma_{j}-\mathfrak{b}_{i}\left(\Delta \gamma_{j}\right)
$$

The numerical approximation of the Jacobian (i.e., $J_{i j}=\frac{d f_{i}}{d \Delta \gamma_{j}} \approx \frac{\delta f_{i}}{\delta \Delta \gamma_{j}}, \forall(i, j) \in \mathcal{N}^{2}$ ) was used for the Newton-Raphson algorithm as it was shown to offer more stability than the analytical evaluation. For completeness, the latter is provided below:

$$
J_{i j}=\mathcal{A}_{i j}+\delta_{i j} \frac{m_{i} \Delta \gamma_{i}^{m_{i}-1}}{\left(\Delta t \dot{\gamma}_{i}^{0}\right)^{m_{i}}}\left[s_{i}+\sum_{k \in \mathcal{N}} h_{i k} \Delta \gamma_{k}\right], \forall(i, j) \in \mathcal{N}^{2}
$$

\section{Results}

\subsection{FEM model}

The constitutive model described above was implemented in a "user material" subroutine VUMAT for the commercial finite element program ABAQUS/EXPLICIT v6.14 (2014). The polycrystalline model with 2,592 cubic elements proposed by Fernández et al. (2011) was used here. In this model, each element represents a full Mg AZ31 alloy crystal with its own crystal lattice orientation. The model crystallographic grain orientation distribution was fitted to the rolled and annealed alloy sheet $<0001>$ texture. Both the normal direction and rolling direction compressions presented by Fernández et al. (2011) were reproduced here by compressing the numerical sample along and perpendicular to the $<0001>$ direction, respectively. It was shown that this approximation actually provides the same degree of accuracy for the stress-strain curves as a more refined model with a Voronoï diagram representation of the polycrystalline aggregate (Fernández et al., 2013). The same rolled sheet initial crystallographic texture and calibrated parameters reached for 
the RI model (Fernández et al., 2011) are adopted here. The parameters specific to the RD formulation, i.e., the strain rate sensitivity coefficients $m_{i}$ and the reference shear rates $\dot{\gamma}_{i}^{0}$ were either obtained from the literature (see Section 4.2.1) or determined so as to ensure compatibility withthe RI formulation (see Section 4.2.2), respectively. The CPFE model was validated against uniaxial compression tests along the normal and rolling directions at high strain rate $\left(\sim 10^{3} s^{-1}\right)$ and at room temperature for a basal fibre texture sample of Mg AZ31 alloy (Ulacia et al., 2010).

\subsection{Parameters space}

\subsubsection{Strain rate sensitivity coefficient}

For cubic metals, the same $m_{i}$ values can be assigned for all systems (Hutchinson, 1976; Asaro and Needleman, 1985; Cuitiño and Ortiz, 1992; Yoon et al., 2007; Zhao et al., 2007; Li et al., 2008). However, due to the high anisotropy of the crystalline structure of hcp metals such as Mg alloys, $m_{i}$ should be defined for each system independently: three basal modes $\langle a\rangle$, three prismatic $\langle a\rangle$, six pyramidal $\langle a\rangle$, six pyramidal $\langle c+a\rangle$ and six tensile twins (Christian and Mahajan, 1995; Bettles and Barnett, 2012). It is widely accepted that basal slip and tensile twin systems' CRSS are independent of strain rate and temperature (Watanabe and Ishikawa, 2009). Consequently, $m_{\text {basal }}=m_{\text {twin }} \approx 0$, but are actually taken as $10^{-6}$, see Table 1 and Appendix A for a justification of this value.

For non-basal slip systems, $m_{i}$ can be approximated by:

$$
m=\frac{d(\log (\tau))}{d(\log (\dot{\varepsilon}))} \approx \frac{\Delta \log (\tau)}{\Delta \log (\dot{\varepsilon})}
$$

where $m$ is the slope of the log-log stress-strain rate curve. $m_{\text {prismatic }}$ is taken here as an average of the values provided by Watanabe and Ishikawa (2009) for different strain rates and temperatures, see Table 1. Following the same approach, $m_{\text {pyramidal }}$ is inferred from the work published by Ulacia et al. (2010). It is widely accepted that pyramidal slips accommodate most of the deformation when a compression along the normal direction is produced (Agnew et al., 2003; Shaffer et al., 2010), thus making this value dramatically important for the behavior of the model. The calculated values were used in all simulations shown here, and thus no calibration procedure for those coefficients was performed.

Table 1: Strain rate sensitivity coefficients $m_{i}$

\begin{tabular}{lcc}
\hline Deformation mechanism & $m_{i}$ & Reference \\
\hline \hline Basal $\langle a\rangle$ & $1 \times 10^{-6}$ & Watanabe and Ishikawa (2009) \\
Prismatic $\langle a\rangle$ & $2.35 \times 10^{-2}$ & Watanabe and Ishikawa (2009) \\
Pyramidal $\langle a\rangle$ & $7.42 \times 10^{-3}$ & Ulacia et al. (2010) \\
Pyramidal $\langle c+a\rangle$ & $7.42 \times 10^{-3}$ & Ulacia et al. (2010) \\
Twin & $1 \times 10^{-6}$ & Watanabe and Ishikawa (2009) \\
\hline
\end{tabular}

\subsubsection{Reference shear rate}

The lack of information about $\dot{\gamma}_{i}^{0}$ suggests this parameter as a "free parameter" of the model which should be calibrated. However, in this work we propose a theoretical approach to obtain a physically grounded reasonable value. This approach consists in finding the value of $\dot{\gamma}^{0}$ such that the RD model fits the RI model for the strain rate (and thus $\dot{\gamma}$ ) at which the quasi-static experiments were done, i.e., $10^{-4} s^{-1}$. To this end, the approach described in Section 4.3 was followed and the explicit model with $T_{\max }=10^{-5} \mathrm{~s}$ was used (see Section 4.4). The calibration was done for the compression in the normal direction and led to $\dot{\gamma}^{0}=10^{-4} s^{-1}$, see Figure 2. This value is reasonable when compared to the values proposed in the literature for other materials and slip systems (Asaro and Needleman, 1985; Cuitiño and Ortiz, 1992; Yoon et al., 2007; Zhao et al., 2007; Li et al., 2008). 


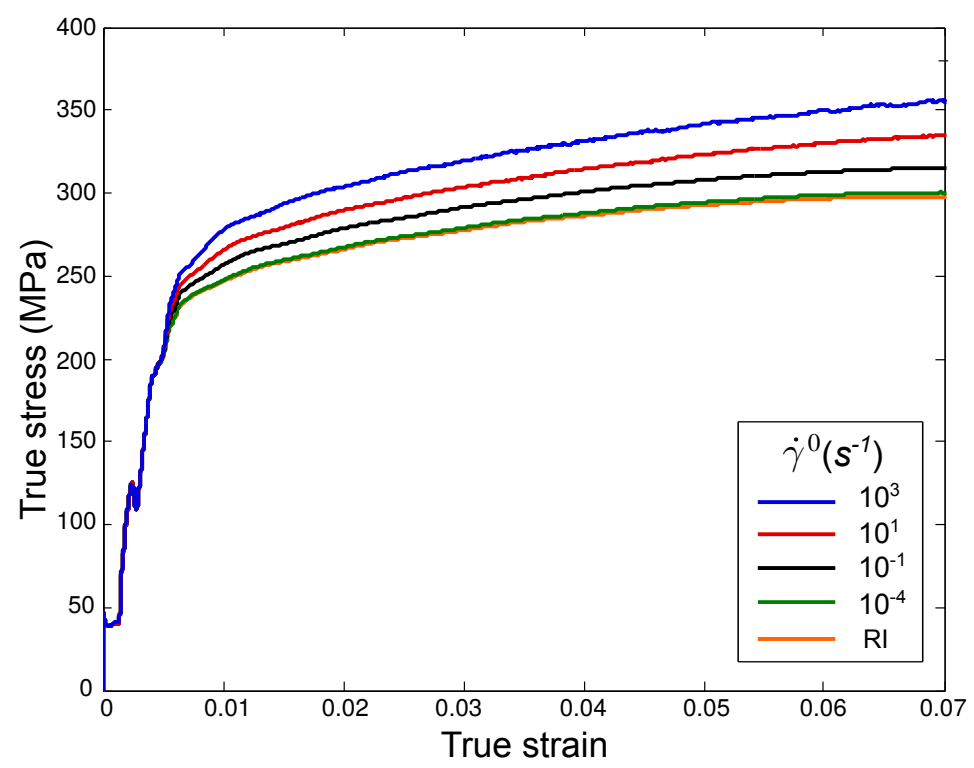

Figure 2: True stress-strain curves for quasi-static $\left(1 \times 10^{-4} s^{-1}\right)$ compression in the normal direction with the RD model for different $\dot{\gamma}^{0}$, and the RI model.

\subsection{Artificial strain rate approach}

The use of an explicit time stepping algorithm for typical RD FEM calculations at strain rate lower than $1 s^{-1}$ invariably leads to unmanageable calculation times. As this model aims at covering all the strain rates larger than $1 \times 10^{-4} s^{-1}$ (for which the RI model can be used), an alternative approach is proposed here for the strain rates situated in the range $10^{-4}-10^{3} s^{-1}$ and for which dynamic (inertia) effects are avoided. As can be seen in Equation (15), the rate effects are actually function of $\frac{\dot{\gamma}}{\dot{\gamma} 0}$. Additionally, in the absence of catastrophic localisation of deformation, $\dot{\gamma}$ can safely be expected to be proportional to the loading macroscopic strain rate $\dot{\varepsilon}$. As a consequence, the same rate effects should be observed if instead increasing or decreasing by a factor $\dot{\varepsilon}$ (and thus $\dot{\gamma}$ ), one would instead increase or decrease, respectively, $\dot{\gamma}^{0}$ by the same factor. As the time step would thus remain unchanged, time-consuming low rate calculations can thus be done by fast higher rate calculations.

Figure 3 confirms the validity of this approach for true compression stress-strain curves for three different strain rates: $10^{3} \mathrm{~s}^{-1}, 10^{2} \mathrm{~s}^{-1}$ and $1 \mathrm{~s}^{-1}$ by either applying the real rate ("Real", $\dot{\varepsilon}$ ) or taking the $10^{3} \mathrm{~s}^{-1}$ simulation and increasing by a factor 10 and $10^{3}$ the value of $\dot{\gamma}^{0}$ ("Artificial", $\dot{\varepsilon}_{\text {art }}$ ). For the particular case of $1 \mathrm{~s}^{-1}$, the simulation with the real compression velocity took several weeks, whereas for the artificial case - setting $\dot{\gamma}^{0}=10^{-1} s^{-1}$ - the simulations took only a couple of hours.

\subsection{Explicit shear window study}

Following Equation (17), the shear window $\tilde{\gamma}$ is related to the current maximum shear rate of the systems $\dot{\gamma}_{\max }$ and the instantaneous time window $T_{\max }$. We show in the following that $\tilde{\gamma}$ is actually constant for all rates, with or without the use of the artificial strain rate (see Section 4.3).

To this end, three compression strain rates were considered for both real $\dot{\varepsilon}$ and artificial $\dot{\varepsilon}_{\text {art }}$ strain rates of: $10^{3} \mathrm{~s}^{-1}, 10^{2} \mathrm{~s}^{-1}$ and $10 \mathrm{~s}^{-1}$. Figure 4 shows the particular case of $\dot{\varepsilon}=10^{3} \mathrm{~s}^{-1}$ for a pyramidal slip system of a) a weighted average of all the elements and of b) one arbitrary element in the centre of polycrystalline sample under normal direction compression. Without the variable moving average algorithm, i.e., $T_{\max }=0$, and using directly the instantaneous explicit approximation of the shear rate, the simulation became unstable and failed. When the time window was modified manually by setting $T_{\max }$ in Equation (17) to an arbitrary small value of $10^{-7} \mathrm{~s}$, the simulation run and completed. However, although the simulation was completed showing slight oscillations for the averaged shear rate of a pyramidal slip system 


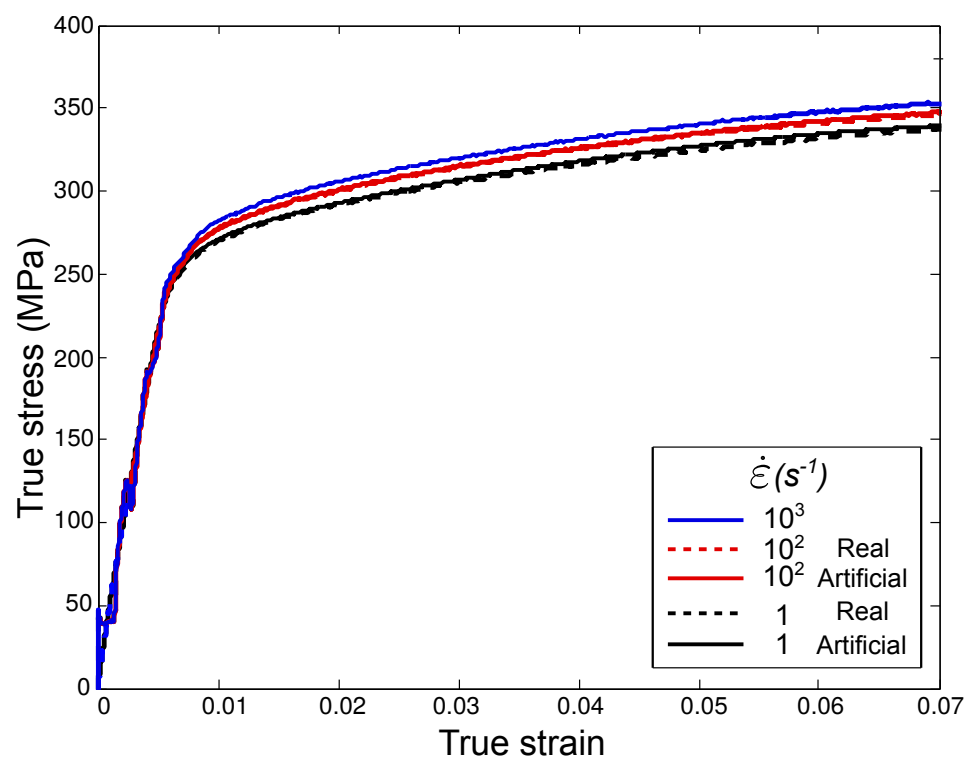

Figure 3: True stress-strain curves for uniaxial compression along the normal direction for different real and artificial strain rates.

for all elements, see green curve in Figure 4(a), when looking at the actual shear rate of the slip system for an arbitrary element in the middle of the domain, strong oscillations with unrealistic values of shear rate were seen, see green curve in Figure 4(b). These dramatic oscillations of shear rates irremediably lead to an unrealistic physical behaviour. In other words, despite the fact that the averaged activity of the overall sample is smooth, the elements are supporting unrealistic shear rate values as a direct consequence of the explicit solver approach ${ }^{2}$. When exploring the other extreme case, i.e., with large time window $T_{\max }=10^{-3} \mathrm{~s}$, the strong oscillations in the system activity at a particular element are smoothed and very similar in both cases, see red curves in Figure 4. However, the activity of the system of the arbitrary element never comes back to zero during the simulation despite a lack of activity as other values for low $T_{\max }$ seem to indicate (probably due to a rotation of this particular crystal). This is actually expected for a large time window because of the averaging "inertia".

The optimal $T_{\max }$ was thus defined as the one that smoothes oscillations at each strain rate regime while allowing for a relatively fast decrease of rate during activity slow down. The values for each rates are reported in Table 2. As can be seen in the table, it was actually found that, although the optimum $T_{\max }$ varies with the loading strain rate, the corresponding shear window was found to be constant with $\tilde{\gamma}=10^{-2}$ independently of the loading rates. Note that for the artificial rates, the $T_{\max }$ value is the same as the one for the underlying simulated rate (as only $\dot{\gamma}^{0}$ is modified, the stochastic slip bursts are indeed expected to remain similar).

Finally, Figure 5 confirms that pyramidal slip averaged behaviours are consistent using either the real or the artificial or artificial rates for several strain rates as long as the optimal value of $T_{\max }$ for each case is taken. Again, this is automatically verified for $\tilde{\gamma}=10^{-2}$.

As a conclusion, the shear window $\tilde{\gamma}$ can actually be optimised for explicit convergence (to $\tilde{\gamma}=1 \times 10^{-2}$ for $\mathrm{Mg}$ AZ31 alloy) independently of the loading rates.

\footnotetext{
${ }^{2}$ Note that the implicit formulation still exhibits relatively large oscillations in the individual local slip shear rates (but much less than the non-averaged explicit formulation). However, the solver controls the local stability to a sufficient extent so that the global behaviour (and thus stress-strain curve) is still reliable and the simulation remains stable (unlike the non-averaged explicit formulation).
} 

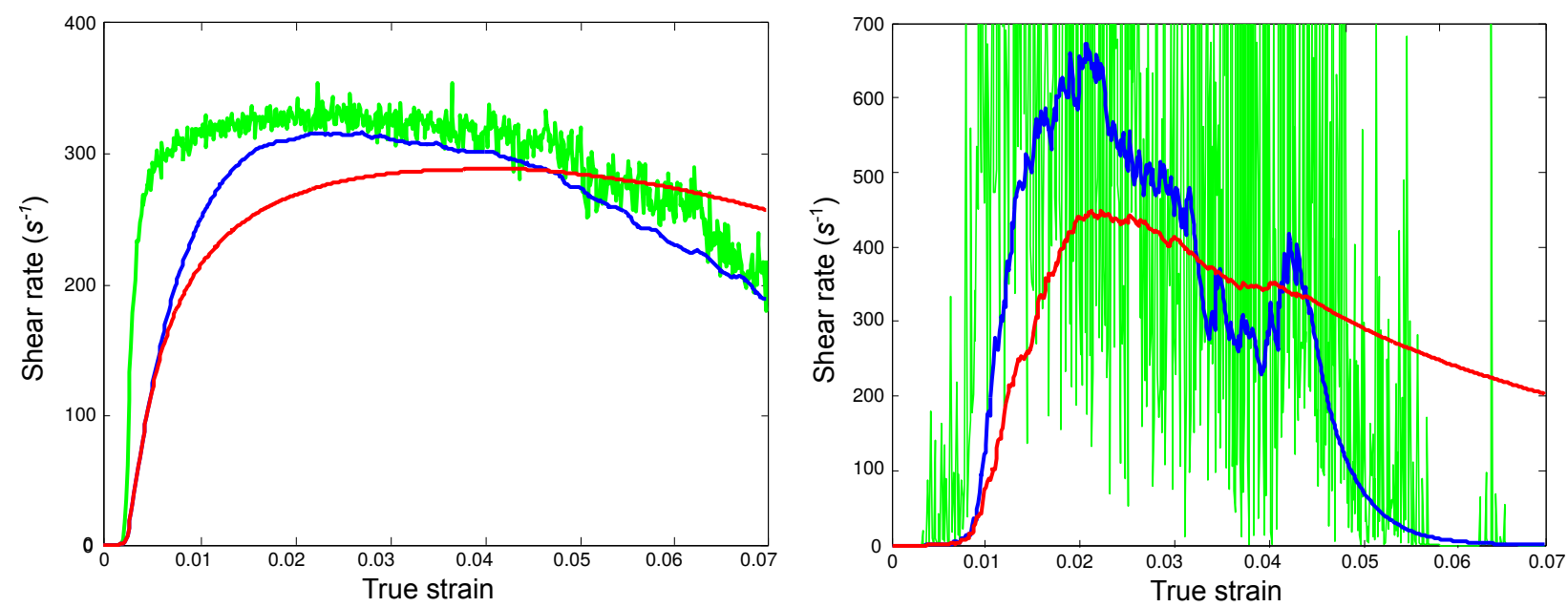

Figure 4: Pyramidal activity study for different $T_{\max }$ windows for $\dot{\varepsilon}=10^{3} \mathrm{~s}^{-1}$ : a) weighted average of a pyramidal slip system activity of all elements in the FEM model, and b) local activity of such system at an arbitrary element in the centre of the FEM model.

Table 2: Optimal time window $T_{\max }$ for several strain rates.

\begin{tabular}{lcccc}
\hline$\dot{\varepsilon}\left(s^{-1}\right)$ & $\dot{\varepsilon}_{\text {art }}\left(s^{-1}\right)$ & $T_{\max }$ & $\dot{\gamma}^{0} s^{-1}$ & $\tilde{\gamma}=\dot{\varepsilon} T_{\max }$ \\
\hline \hline 1000 & 1000 & $1 \times 10^{-5}$ & $1 \times 10^{-4}$ & $1 \times 10^{-2}$ \\
100 & - & $1 \times 10^{-4}$ & $1 \times 10^{-4}$ & $1 \times 10^{-2}$ \\
1000 & 100 & $1 \times 10^{-5}$ & $1 \times 10^{-3}$ & $1 \times 10^{-2}$ \\
10 & - & $1 \times 10^{-3}$ & $1 \times 10^{-4}$ & $1 \times 10^{-2}$ \\
1000 & 10 & $1 \times 10^{-5}$ & $1 \times 10^{-2}$ & $1 \times 10^{-2}$ \\
\hline
\end{tabular}

\subsection{Validation}

In this subsection, both resolution approaches, explicit and implicit, are used to verify the ability of the CPFE model to describe Mg AZ31 alloy deformation mechanisms under slip or twin dominated deformations at high strain rates. The test conditions were described in Section 4.1. Figures 6 and 7 show the numerical and experimental true stress-strain results for compression tests along the normal and rolling directions, respectively. In particular, the dash line corresponds to experimental results published by Ulacia et al. (2010), the continuum black line represents the simulated RI results obtained by Fernández et al. (2011), and the blue and red lines correspond to the simulated RD results at a strain rate of $10^{3} \mathrm{~s}^{-1} \mathrm{using}$ the explicit and implicit schemes, respectively. Note finally that the explicit simulations were done with a smaller time step to ensure convergence.

Figures 6 and 7 show a relatively good correspondence between simulations and experimental results for both proposed integration algorithms, explicit and implicit. The lack of rate dependency for the basal slip and twin dominated portions of the deformation in Figure 7, i.e., the initial yielding and the first plateau, as well as the rate dependency of the pyramidal slip dominated portions of the deformation in Figure 7, i.e., the subsequent hardening, along with the full deformation in Figure 6 are perfectly captured. The model is thus able to simulate the overall mechanical response of the Mg AZ31 alloy at dynamic strain rates for normal and rolling direction compressions. It is worth noting that discrepancies appear when pyramidal $\langle c+a\rangle$ slips operate in combination with basal slips. For example, in Figure 7 which reveals the usual concave flow stress curve commonly produced in $\mathrm{Mg}$ alloys by the activation of twinning at the early stages of the deformation 


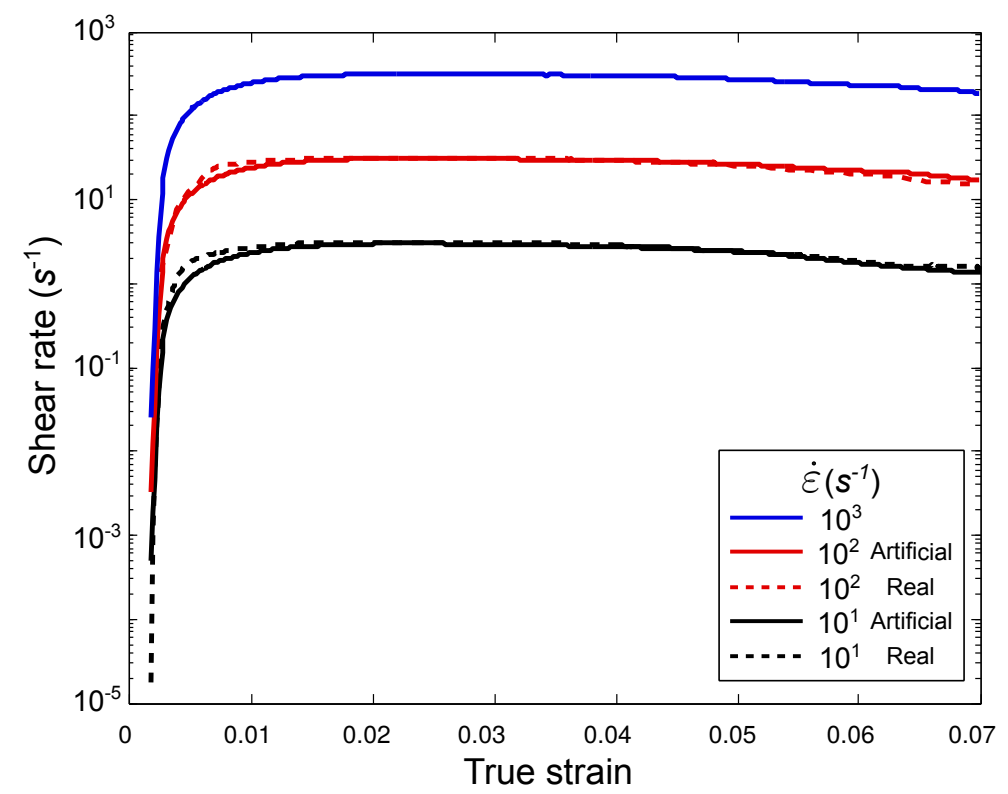

Figure 5: Average shear rate activity for a pyramidal system of all elements in the FEM model at different real and artificial strain rates.

for compression along the rolling direction, differences are observed during the last deformation stages when the strain is mainly accommodated by these slip systems. This also occurs when a compression along normal direction is performed, as seen in Figure 6. The fit could thus be possibly enhanced by recalibrating the corresponding RI interaction parameters, or some RI parameters that did not have much importance in the quasi-static cases (e.g., prismatic systems), and which might play a more important role at high rate, but the experimental lack of repeatability in the literature potentially defeats the purpose of such effort. It is finally remarkable that no calibration (except the one related to the numerical stability of the explicit scheme) was performed to achieve these results.

\section{Discussion}

The implementation of a strain rate dependence law and the explicit and implicit resolutions of the resulting constitutive model, see Equation (25), have required the implementation of novel approaches to ensure a) a realistic behaviour of the explicit implementation, and b) a manageable calculation time for low strain rates. Both explicit and implicit numerical approaches were shown to capture the intrinsic mechanisms of the differentiated rate dependencies of slip and twin systems in Mg AZ31 alloys. It is noticeable that these results were obtained without any previous calibration, with the strain rate sensitivity coefficients $m_{i}$ taken from the literature and the reference strain rates determined in Section 4.2.2 so as to ensure compatibility between the RI and RD models. The use of this model for other hcp alloys is straightforward and only requires the corresponding initial texture and the values for the strain rate sensitivity coefficients and the shear reference strain rates.

The new constitutive model presented here is very versatile. The variable moving average proposed for the explicit scheme paves the road to many different scenarios where the resultant system of equations might be ill-conditioned, e.g., other hcp alloys or additional considerations of temperature dependency. It is also remarkable that the shear window $\tilde{\gamma}$ was found to be constant for independently of the loading rate. Future studies should explore the physical significance of this conclusion, as it could possibly be (loosely) related to dislocation burst volume dependency. Finally, an artificial strain rate approach was proposed to avoid long calculation times in low rates simulations. 


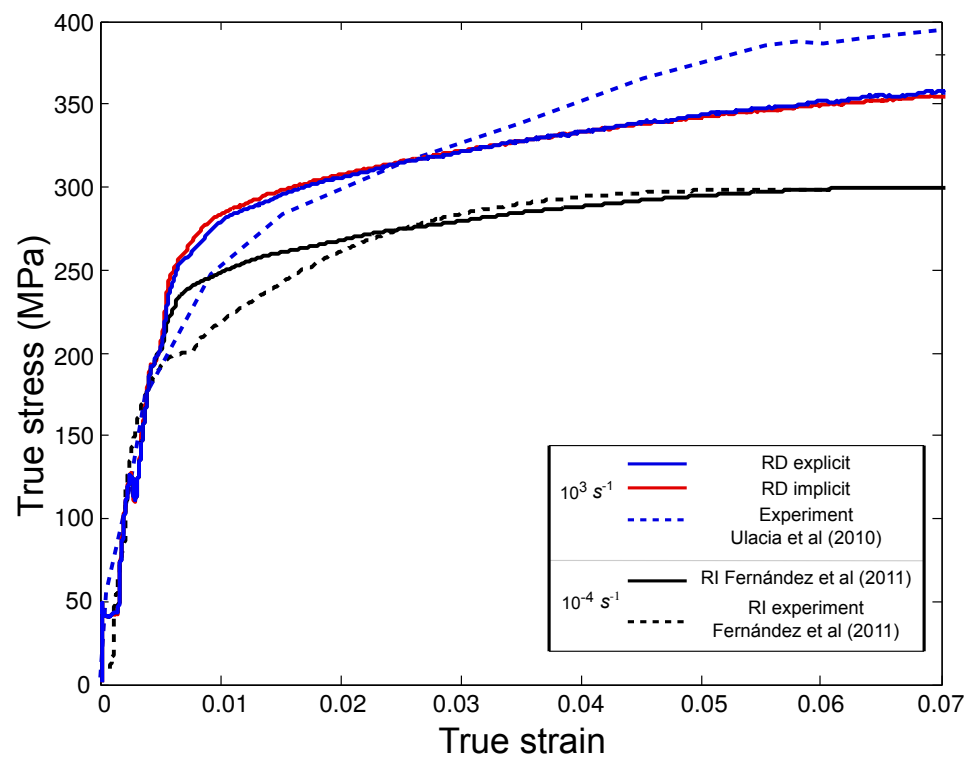

Figure 6: Simulated and experimental true stress-strain curves for uniaxial compression along the normal direction at high strain rate $\left(10^{3} s^{-1}\right)$.

Some discrepancies between experimental and simulations true stress-true strain curves were observed. However, the lack of experimental repeatability is shown to lead to important discrepancies in the stressstrain curves of the same alloy (Watanabe and Ishikawa, 2009; Ulacia et al., 2010; Li, 2012; Ahmad and Shu, 2014). Additionally, if all published works reveal the same trends in their results, values of CRSSs, hardening parameters, twinning volume fraction, etc. are slightly different between them, potentially due to initial microstructural features which affect directly the mechanical behavior. The influence of these features is probably more relevant at high strain rates, thus explaining the large variability in the resulting stress-strain curves. Finally, it has been observed that during compression along the normal direction at high strain rates, tensile twinning occurs even in unfavourably oriented grains (Dudamell et al., 2011). For compression along the rolling direction at high strain rates, it has been shown that the hardening rate is lower than in the quasi-static case (Dudamell et al., 2011). This suggests that more mechanisms should be included in the CPFE model in order to capture more precisely some of the intrinsic mechanisms of high rate alloy deformation.

\section{Conclusion}

A new strain rate dependent CPFE model was presented here for hcp metals. The model was validated against experimental results for the $\mathrm{Mg}$ AZ31 alloy at room temperature, confirming the ability of the model to capture both low and high rate behaviours with a unique set of material parameters. Whereas the stability and convergence were readily demonstrated for the implicit formulation, the development of a novel variable moving average methodology was needed for the explicit formulation, thus avoiding the more expensive implicit direct resolution of the system of nonlinear equations - ill-conditioned in this case. Finally, an artificial rate approach was proposed to reduce the time calculation of the lower rate loading simulations when no inertial effects are expected. To the best of knowledge of the authors, it is the first time that a RD law with realistic rate dependency exponents is successfully implemented for hcp crystals in a CPFE model. 


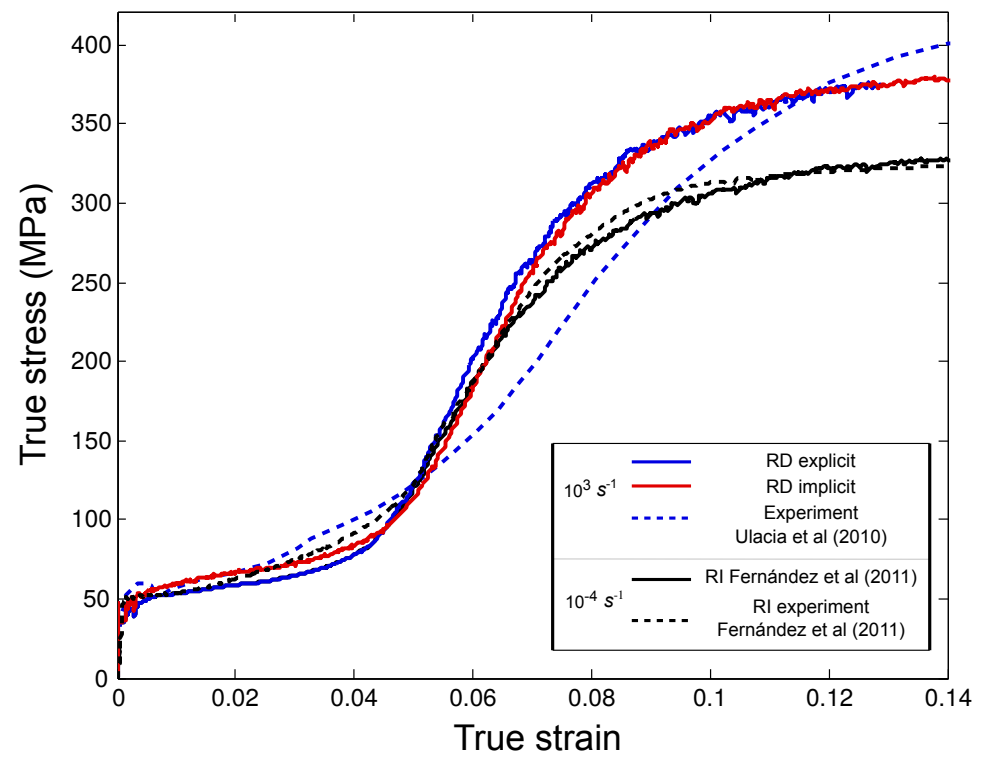

Figure 7: Simulated and experimental true stress-strain curves for uniaxial compression along the rolling direction at high strain rate $\left(10^{3} s^{-1}\right)$.

\section{Acknowledgements}

The authors are grateful to Dr. Yujie Wei, Dr. Teresa Pérez-Prado and Dr. Nathamar Dudamell for very productive discussions. A.F. and A.J. would like to thank the vehicle interior manufacturer, Grupo Antolin Ingenieria, S.A., within the framework of the project MAGNO2008-1028-CENIT Project funded by the Spanish Ministry. Funding from the ESTRUMAT-S2009/MAT-1585 grant (Madrid Regional Government) is acknowledged. A.J. also acknowledges support from the Juan de la Cierva grant from the Spanish Ministry of Science and Innovation and from the Amarout grant from the European Union. Finally, the authors would like to thank the computer resources from the Oxford Supercomputing Centre at the University of Oxford.

\section{Appendix A. Strain rate sensitivity coefficient study for basal slip and twin systems}

The results presented in this appendix were carried out with the implicit solver proposed in this work for the case of compression along the rolling direction. This choice is motivated by the fact that all deformation mechanisms are activated at some point during the simulation.

This study is aimed at studying the influence of $m_{\text {basal }}$ and $m_{\text {twin }}$ on the stability of the system. Based on the literature, both mechanisms are strain rate and temperature independent (Watanabe and Ishikawa, 2009). However, from a numerical standpoint, a small but negligible rate dependent contribution can potentially help to homogenise the resultant system of nonlinear equations obtained in Equation (15). In this equation, if $m_{\text {basal }}$ and $m_{\text {twin }}$ are set to zero, the presence of the non-zero rate contribution of the pyramidal and prismatic systems in the system of 24 nonlinear equations can be expected to be highly heterogeneous. In such case, reaching the correct solution can become a challenging task. The goal of this study is to evaluate how "far" from zero $m_{\text {basal }}$ and $m_{\text {twin }}$ can be without modifying the overall mechanical behaviour of the material. As $m_{\text {basal }}$ and $m_{\text {twin }}$ become larger, the system of equations become more homogeneous. In this study the reference shear strain rates $\dot{\gamma}_{i}^{0}$ are set to $10^{-4} \mathrm{~s}^{-1}$ for all systems.

Figure A.8 shows the results for several $m_{\text {basal }}$ and $m_{\text {twin }}$ values. If both $m_{\text {basal }}$ and $m_{\text {twin }}$ are set to $10^{-3}$ or smaller, the stress-strain curve remains quasi-identical. Although the true stress-strain curves look similar for values increasingly lower than $10^{-3}$ (including $m_{\text {basal }}=m_{\text {twin }}=0$ ), as this value decreased, the nonlinear solver could increasingly not reach a solution for a number of steps and elements during the 
simulation. Therefore, a compromise of $m_{\text {basal }}=m_{\text {twin }}=1 \times 10^{-6}$ was taken to guarantee the physical requirement of both systems, while mathematically homogenising the system (and thus minimising the number of non-converged cases).

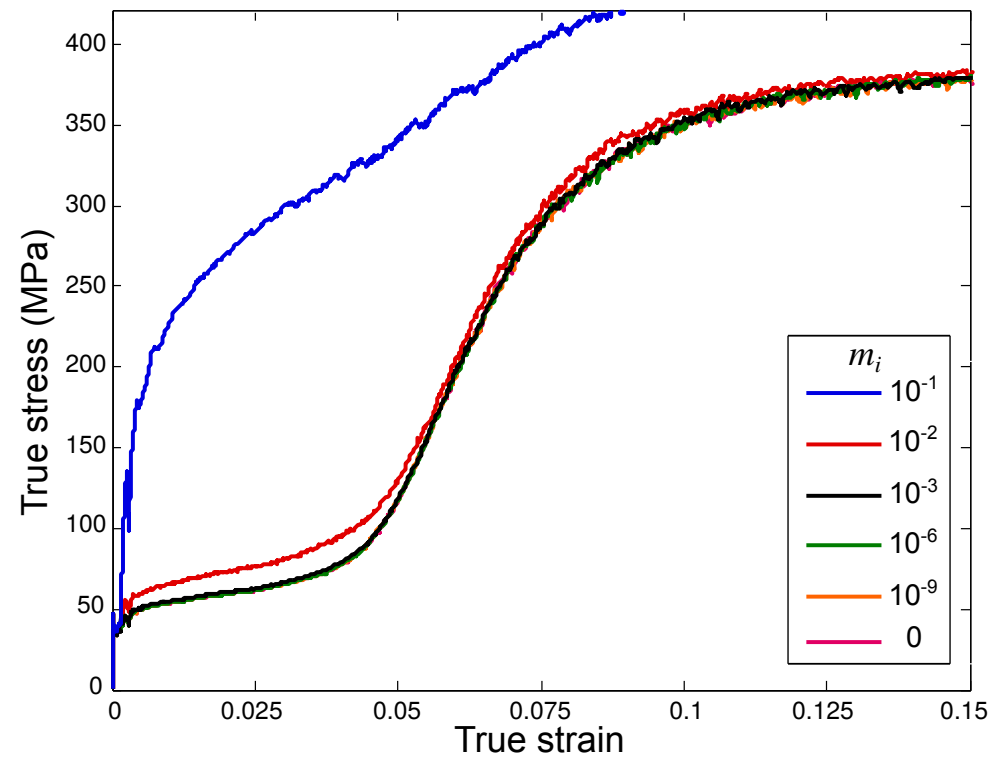

Figure A.8: Sensibility study of $m_{\text {basal }}$ and $m_{\text {twin }}$ in the case of uniaxial compression along the rolling direction at high strain rate $\left(1 \times 10^{3} s^{-1}\right)$.

\section{References}

ABAQUS/EXPLICIT v6.14, 2014. User's Manual ABAQUS Inc Richmond USA.

Abdolvand, H., Daymond, M. R., 2013a. Multi-scale modeling and experimental study of twin inception and propagation in hexagonal close-packed materials using a crystal plasticity finite element approach - Part I: Average behavior. Journal of the Mechanics and Physics of Solids 61, $783-802$.

Abdolvand, H., Daymond, M. R., 2013b. Multi-scale modeling and experimental study of twin inception and propagation in hexagonal close-packed materials using a crystal plasticity finite element approach - Part II: Local behavior. Journal of the Mechanics and Physics of Solids 61, $803-818$.

Aghion, E., Bronfin, B., Eliezer, D., 2001. The role of the magnesium industry in protecting the environment. Journal of Materials Processing Technology 117, $381-385$.

Agnew, S. R., Duygulu, O., 2005. Plastic anisotropy and the role of non-basal slip in magnesium alloy AZ31B. International Journal of Plasticity 21 (6), $1161-1193$.

Agnew, S. R., Tomé, C. N., Brown, D. W., Holden, T. M., Vogel, S. C., 2003. Study of slip mechanisms in a magnesium alloy by neutron diffraction and modeling. Scripta Materialia 48 (8), $1003-1008$.

Agnew, S. R., Yoo, M. H., Tomé, C. N., 2001. Application of texture simulation to understanding mechanical behavior of Mg and solid solution alloys containing Li or Y. Acta Materialia 49 (20), $4277-4289$.

Ahmad, I. R., Shu, D. W., 2014. Compressive and constitutive analysis of az31b magnesium alloy over a wide range of strain rates. Materials Science and Engineering A 592, $40-49$.

Al-Samman, T., Gottstein, G., 2008. Room temperature formability of a magnesium AZ31 alloy: Examining the role of texture on the deformation mechanisms. Materials Science and Engineering A 488 (1-2), $406-414$.

Anand, L., 2004. Single-crystal elasto-viscoplasticity: application to texture evolution in polycrystalline metals at large strains. Computer Methods in Applied Mechanics and Engineering 193, 5359 - 5383.

Anand, L., Kothari, M., 1996. A computational procedure for rate-independent crystal plasticity. Journal of the Mechanics and Physics of Solids 44 (4), $525-558$.

Armstrong, R. W., Walley, S. M., 2008. High strain rate properties of metals and alloys. International Materials Reviews 53 (3), $105-128$.

Asaro, R. J., Needleman, A., 1985. Texture development and strain hardening in rate dependent polycrystals. Acta Metallurgica $33,923-953$.

Barnett, M. R., 2003. A taylor model based description of the proof stress of magnesium AZ31 during hot working. Metallurgical and Materials Transactions A 34 (9), $1799-1806$. 
Barnett, M. R., 2007a. Twinning and the ductility of magnesium alloys Part I: "Tension" twins. Materials Science and Engineering A 464 (1-2), $1-7$.

Barnett, M. R., 2007b. Twinning and the ductility of magnesium alloys Part II: "Contraction" twins. Materials Science and Engineering 464, $8-16$.

Barnett, M. R., Keshavarz, Z., Beer, A. G., Atwell, D., 2004. Influence of grain size on the compressive deformation of wrought Mg-3Al-1Zn. Acta Materialia 52 (17), 5093 - 5103.

Bettles, C., Barnett, M. R., 2012. Advances in wrought magnesium alloys. Cambridge: Woodhead Publishing.

Beyerlein, I. J., McCabe, R. J., Tomé, C. N., 2011. Effect of microstructure on the nucleation of deformation twins in polycrystalline high-purity magnesium: A multi-scale modeling study. Journal of Mechanics and Physics of Solids 59, 988 1003

Bilby, B. A., Crocker, A. G., 1965. The theory of the crystallography of deformation twinning. Proceedings of The Royal Society $288,240-255$.

Chapuis, A., Driver, J. H., 2011. Temperature dependency of slip and twinning in plane strain compressed magnesium single crystals. Acta Materialia 59, 1986 - 1994.

Chin, G. Y., Mammel, W. L., 1970. Competition among basal, prism, and pyramidal slip modes in hcp metals. Metallurgical Transactions 1, $357-361$.

Choi, S. H., Kim, D. H., Lee, H. W., Seong, B. S., Piao, K., Wagoner, R., 2009. Evolution of the deformation texture and yield locus shape in an az31 mg alloy sheet under uniaxial loading. Materials Science and Engineering A 526, 38 - 49.

Choi, S. H., Kim, D. H., Lee, H. W., Shin, E. J., 2010a. Simulation of texture evolution and macroscopic properties in Mg alloys using the crystal plasticity finite element method. Materials Science and Engineering A 527 (4-5), 1151 - 1159.

Choi, S. H., Kim, D. H., Park, S. S., You, B. S., 2010b. Simulation of stress concentration in Mg alloys using the crystal plasticity finite element method. Acta Materialia 58, $320-329$.

Choi, S. H., Kim, D. W., Seong, B. S., Rollett, A. D., 2011. 3-d simulation of spatial stress distribution in an AZ31 Mg alloy sheet under in-plane compression. International Journal of Plasticity 27, $1702-1720$.

Choi, S. H., Shin, E. J., Seong, B. S., 2007. Simulation of deformation twins and deformation texture in an az31 mg alloy under uniaxial compression. Acta Materialia 55, $4181-4192$.

Christian, J. W., Mahajan, S., 1995. Deformation twinning. Progress in Materials Science 39 (1-2), $1-157$.

Couling, S. L., Pashak, J. F., Sturkey, L., 1959. Unique deformation and aging characteristics of certain magnesium-based alloys. Transactions of the American Society of Metals 51, $94-107$.

Couret, A., Caillard, D., 1985. An in situ study of prismatic glide in magnesium-i. the rate controlling mechanism. Acta Metallurgica $33(8), 1447-1454$.

Cuitiño, A. M., Ortiz, M., 1992. Computational modelling of single crystals. Modelling and Simulation in Materials Science and Engineering 1, $225-263$.

del Valle, J. A., Ruano, O. A., 2007. Separate contributions of texture and grain size on the creep mechanisms in a fine-grained magnesium alloy. Acta Materialia 55 (2), $455-466$.

Dudamell, N. V., Ulacia, I., Gálvez, F., Yi, S., Bohlen, J., Letzig, D., Hurtado, I., Pérez-Prado, M. T., 2011. Twinning and grain subdivision during dynamic deformation of a Mg AZ31 sheet alloy at room temperature. Acta Materialia 59, $6949-$ 6962.

Dudamell, N. V., Ulacia, I., Gálvez, F., Yi, S., Bohlen, J., Letzig, D., Hurtado, I., Pérez-Prado, M. T., 2012. Influence of texture on the recrystallization mechanisms in an AZ31 mg sheet alloy at dynamic rates. Materials Science and Engineering A $532,528-535$.

Easton, M., Beer, A., Barnett, M., Davies, C., Dunlop, G., Durandet, Y., Blacket, S., Hilditch, T., Beggs, P., 2008. Magnesium alloy applications in automotive structures. JOM Journal of the Minerals, Metals and Materials Society 60 (11), 57 - 62.

El-Magd, E., Abouridouane, M., 2003. Influence of strain rate and temperature on the compressive ductility of Al, Mg and Ti alloys. Journal de Physique Archives IV France 110, 15 - 20.

El-Magd, E., Abouridouane, M., 2006. Characterization, modelling and simulation of deformation and fracture behaviour of the light-weight wrought alloys under high strain rate loading. International Journal of Impact Engineering $32,741-758$.

Fernández, A., Jérusalem, A., Gutiérrez-Urrutia, I., Pérez-Prado, M. T., 2013. Three-dimensional investigation of grain boundarytwin interactions in a $\mathrm{mg}$ az31 alloy by electron backscatter diffraction and continuum modeling. Acta Materialia 61 , $7679-7692$

Fernández, A., Pérez-Prado, M. T., Wei, Y., Jérusalem, A., 2011. Continuum modeling of the response of a Mg alloy AZ31 rolled sheet during uniaxial deformation. International Journal of Plasticity 27, 1739 - 1757.

Friedrich, H., Schumann, S., 2001. Research for a "new age of magnesium" in the automotive industry. Journal of Materials Processing Technology 117, $276-281$.

Gao, C. Y., Zhang, L. C., Guo, W. G., Li, Y. L., Lu, W. R., Ke, Y. L., 2014. Dynamic plasticity of AZ31 magnesium alloy: Experimental investigation and constitutive modeling. Materials Science and Engineering A $613,379-389$.

Gehrmann, R., Frommert, M. M., Gottstein, G., 2005. Texture effects on plastic deformation of magnesium. Materials Science and Engineering A 395 (1-2), $338-349$.

Graff, S., Brocks, W., Steglich, D., 2007. Yielding of magnesium: From single crystal to polycrystalline aggregates. International Journal of Plasticity 23, $1957-1978$

Herrera-Solaza, V., LLorca, J., Dogan, E., Karaman, I., Segurado, J., 2014. An inverse optimization strategy to determine single crystal mechanical behavior from polycrystal tests: Application to AZ31 mg alloy. International Journal of Plasticity $57,1-15$.

Hirsch, J., Al-Samman, T., 2013. Superior light metals by texture engineering: optimized aluminum and magnesium alloys for automotive applications. Acta Materialia 61, $818-843$. 
Houtte, P. V., Kanjarla, A. K., Bael, A. V., Seefeldt, M., Delannay, L., 2006. Multiscale modelling of the plastic anisotropy and deformation texture of polycrystalline materials. European Journal of Mechanics A/Solids $25,634-648$.

Hutchinson, B., Barnett, M. R., Ghaderi, A., Cizek, P., Sabirov, I., 2009. Deformation modes and anisotropy in magnesium alloy AZ31. International Journal of Materials Research, 556 - 563.

Hutchinson, J. W., 1976. Bounds and seelf-consistent estimates for creep of polycrystalline materials. Proceedings of the Royal Society of London 348, $101-127$, http://www.jstor.org/stable/79120.

Ishikawa, K., Watanabe, H., Mukai, T., 2005a. High strain rate deformation behavior of an AZ91 magnesium alloy at elevated temperatures. Materials letters 59, $1511-1515$.

Ishikawa, K., Watanabe, H., Mukai, T., 2005b. High temperature compressive properties over a wide range of strain rates in an AZ31 magnesium alloy. Journal of Materials Science 40, 1577 - 1582.

Izadbakhsh, A., Inal, K., Mishra, R., Niewczas, M., 2011. New crystal plasticity constitutive model for large strain deformation in single crystals of magnesium. Computational Materials Science 50, $2185-2202$.

Jain, A., Duygulu, O., Brown, D. W., Tomé, C. N., Agnew, S. R., 2008. Grain size effects on the tensile properties and deformation mechanisms of a magnesium alloy, AZ31B, sheet. Materials Science and Engineering A 486 (1-2), 545 - 555.

Jérusalem, A., Dao, M., Suresh, S., Radovitzky, R., 2008. Three-dimensional model of strength and ductility of polycrystalline copper containing nanoscale twins. Acta Materialia 56, $4647-4657$.

Johnson, G. R., Cook, W. H., 1985. Fracture characteristics of three metals subjected to various strains, strain rates, temperatures and pressures. Engineering Fracture Mechanics 21 (1), $31-48$.

Juan, P.-A., Berbenni, S., Barnett, M. R., Tomé, C. N., Capolungo, L., 2014. A double inclusion homogenization scheme for polycrystals with hierarchal topologies: application to twinning in $\mathrm{mg}$ alloys. International Journal of Plasticity 60 , 182 196.

Kabirian, F., Khan, A. S., Gnupel-Herlod, T., 2015. Visco-plastic modeling of mechanical responses and texture evolution in extruded $\{$ AZ31\} magnesium alloy for various loading conditions. International Journal of Plasticity 68 (0), $1-20$.

Kelley, E. W., Hosford, W. F., 1968. The deformation characteristics of textured magnesium. Transactions of the metallurgical society of AIME 242, $654-661$.

Keshavarz, Z., Barnett, M. R., 2006. EBSD analysis of deformation modes in Mg-3Al-1Zn. Scripta Materialia 55 (10), 915 918.

Khan, A. S., Pandey, A., Gnupel-Herold, T., Mishra, R. K., 2011. Mechanical response and texture evolution of \{AZ31\} alloy at large strains for different strain rates and temperatures. International Journal of Plasticity 27 (5), $688-706$.

Kocks, U. F., Westlake, D. G., 1967. The importance of twinning for the ductility of CPH polycrystals. Transactions of the metallurgical society of AIME 239, 1107 - 1109.

Koike, J., Kobayashi, T., Mukai, T., Watanabe, H., Suzuki, M., Maruyama, K., Higashi, K., 2003. The activity of non-basal slip systems and dynamic recovery at room temperature in fine-grained AZ31B magnesium alloys. Acta Materialia 51 (7), $2055-2065$.

Kuchnicki, S. N., no, A. M. C., Radovitzky, R. A., 2006. Efficient and robust constitutive integrators for single-crystal plasticity modeling. International Journal of Plasticity 22, $1988-2011$.

Kuchnicki, S. N., Radovitzky, R. A., no, A. M. C., 2008. An explicit formulation for multiscale modeling of bcc metals. International Journal of Plasticity 24 (12), $2173-2191$.

Lee, E. H., 1969. Elastic plastic deformation at finite strain. Journal of Applied Mechanics $36,1-6$.

Lee, M. G., Lim, H., Adams, B. L., Hirth, J. P., Wagoner, R. H., 2010. A dislocation density-based single crystal constitutive equation. International Journal of Plasticity 26, $925-938$.

Lévesque, J., Inal, K., Neale, K. W., Mishra, R. K., 2010. Numerical modeling of formability of extruded magnesium alloy tubes. International Journal of Plasticity 26, $65-83$.

Li, H. W., Yang, H., Sun, Z. C., 2008. A robust integration algorithm for implementing rate dependent crystal plasticity into explicit finite element method. International Journal of Plasticity 24, 267-288.

Li, Q. Z., 2012. Mechanical properties and microscopic deformation mechanism of polycrystalline magnesium under high-strainrate compressive loadings. Materials Science and Engineering A 540, $130-134$.

Ling, X., Horstemeyer, M. F., Potirniche, G. P., 2005. On the numerical implementation of 3D rate-dependent single crystal plasticity formulations. International Journal for Numerical Methods in Engineering 63, $548-568$.

Liu, Z. L., Zhuang, Z., Liu, X. M., Zhao, X. C., Zhang, Z. H., 2011. A dislocation dynamics based higher-order crystal plasticity model and applications on confined thin-film plasticity. International Journal of Plasticity $27(2), 201-216$.

Lou, X. Y., Li, M., Boger, R. K., Agnew, S. R., Wagoner, R. H., 2007. Hardening evolution of AZ31B Mg sheet. International Journal of Plasticity $23(1), 44-86$.

Malvern, L., 1969. Introduction to the mechanics of a continuum medium. Prentice-Hall.

Mayama, T., Aizawa, K., Tadano, Y., Kuroda, M., 2009. Influence of twinning deformation and lattice rotation on strength differential effect in polycrystalline pure magnesium with rolling texture. Computational Materials Science $47,448-455$.

Mayeur, J. R., McDowell, D. L., 2007. A three-dimensional crystal plasticity model for duplex Ti-6Al-4V. International Journal of Plasticity 23, $1457-1485$.

McGinty, R. D., McDowell, D. L., 2006. A semi-implicit integration scheme for rate independent finite crystal plasticity. International Journal of Plasticity 22, $996-1025$.

Mordike, B. L., Ebert, T., 2001. Magnesium: Properties - applications - potential. Materials Science and Engineering A 302 (1), $37-45$.

Mukai, T., Mohri, T., Mabuchi, M., Nakamura, M., Ishikawa, K., Higashi, K., 1998. Experimental study of a structural magnesium alloy with high absorption energy under dynamic loading. Scripta Materialia 39, 1249 - 1253.

Nemat-Nasser, S., Obata, M., 1986. Rate-dependent, finite elasto-plastic deformation of polycrystals. Proceedings of the Royal 
Society of London. Series A, Mathematical and Physical Sciences 407, $343-375$.

Pandey, A., Kabirian, F., Hwang, J.-H., Choi, S.-H., Khan, A. S., 2015. Mechanical responses and deformation mechanisms of an $\{$ AZ31\} mg alloy sheet under dynamic and simple shear deformations. International Journal of Plasticity 68 (0), $111-$ 131.

Peirce, D., Asaro, R. J., Needleman, A., 1982. An analysis of nonuniform and localized deformation in ductile single crystals. Acta Metallurgica 30, $1087-1119$.

Peirce, D., Asaro, R. J., Needleman, A., 1983. Material rate dependence and localized deformation in crystalline solids. Acta Metallurgica 31, $1951-1976$.

Prakasha, A., Weygand, S. M., Riedel, H., 2009. Modeling the evolution of texture and grain shape in Mg alloy AZ31 using the crystal plasticity finite element method. Computational Materials Science 45, $744-750$.

Press, W. H., 1992. Numerical recipes for Fortran 77. Cambridge University Press.

Rossiter, J., Brahme, A., Simha, M. H., Inal, K., Mishra, R., 2010. A new crystal plasticity scheme for explicit time integration codes to simulate deformation in 3D microstructures: Effects of strain path, strain rate and thermal softening on localized deformation in the aluminum alloy 5754 during simple shear. International Journal of Plasticity 26 (12), $1702-1725$.

Roters, F., Eisenlohr, P., Hantcherli, L., Tjahjanto, D. D., Bieler, T. R., Raabe, D., 2010. Overview of constitutive laws, kinematics, homogenization and multiscale methods in crystal plasticity finite-element modeling: Theory, experiments, applications. Acta Materialia 58 (4), $1152-1211$.

Serra, A., Bacon, D. J., 2005. Modelling the motion of $11 \overline{2} 2$ twinning dislocations in the HCP metals. Materials Science and Engineering A 400-401, 496 - 498.

Shaffer, J. B., Knezevic, M., Kalidindi, S. R., 2010. Building texture evolution networks for deformation processing of polycrystalline fcc metals using spectral approaches: Applications to process design for targeted performance. International Journal of Plasticity 26, $1183-1194$.

Staroselsky, A., Anand, L., 2003. A constitutive model for hcp materials deforming by slip and twinning: application to magnesium alloy AZ31B. International Journal of Plasticity 19 (10), $1843-1864$.

Staroselsky, A. V., 1998. Crystal plasticity due to slip and twinning. Ph.D. thesis, Massachusetts Institute of Technology.

Sung, J. H., Kim, J. H., Wagoner, R. H., 2010. A plastic constitutive equation incorporating strain, strain-rate, and temperature. International Journal of Plasticity 26 (12), $1746-1771$.

Tang, W., Zhang, S., Peng, Y., Li, D., 2009. Simulation of magnesium alloy AZ31 sheet during cylindrical cup drawing with rate independent crystal plasticity finite element method. Computational Materials Science 46 (2), $393-399$.

Thamburaja, P., 2010. A finite-deformation-based phenomenological theory for shape-memory alloys. International Journal of Plasticity 26, $1195-1219$.

Tucker, M. T., Horstemeyer, M. F., Gullett, P. M., El-Kadiri, H., Whittington, W. R., 2009. Anisotropic effects on the strain rate dependence of a wrought magnesium alloy. Scripta Materialia 60, $182-185$.

Ulacia, I., Dudamell, N. V., Gálvez, F., Yi, S., Prado, M. T. P., Hurtado, I., 2010. Mechanical behavior and microstructural evolution of a Mg AZ31 sheet at dynamic strain rates. Acta Materialia 58, $2988-2998$.

Vagaralia, S. S., Langdon, T. G., 1981. Deformation mechanisms in h.c.p. metals at elevated temperatures - I. creep behavior of magnesium. Acta Metallurgica 29 (12), 1969 - 1982.

Watanabe, H., Ishikawa, K., 2009. Effect of texture on high temperature deformation behavior at high strain rates in a Mg3Al-1Zn alloy. Materials Science and Engineering A 523, $304-311$.

Watanabe, I., Setoyama, D., Iwata, N., Nakanishi, K., 2010. Characterization of yielding behavior of polycrystalline metals with single crystal plasticity based on representative characteristic length. International Journal of Plasticity $26,570-585$.

Watarai, H., 2006. Trend of research and development for magnesium alloys - reducing the weight of structural materials in motor vehicles. Journal of Materials Processing Technology 7, 84-97.

Yoo, M. H., 1981. Slip, twinning, and fracture in hexagonal close-packed metals. Metallurgical Transactions 12A, $409-418$.

Yoo, M. H., Lee, J. K., 1991. Deformation twinning in h.c.p. metals and alloys. Philosophical Magazine A 63, $987-1000$.

Yoon, J. H., Huh, H., Lee, Y. S., 2007. Finite element analysis of polycrystalline deformation with the rate-dependent crystal plasticity. AIP Conference Proceedings 908, $1325-1330$.

Zamiri, A. R., Pourboghrat, F., 2010. A novel yield function for single crystals based on combined constraints optimization. International Journal of Plasticity 26, $731-746$.

Zerilli, F. J., Armstrong, R. W., 1987. Dislocationmechanicsbased constitutive relations for material dynamics calculations. Journal of Applied Physics 61, 1816 - 1825.

Zhang, J., Yu, Q., Jiang, Y. Y., Li, Q. Z., 2011. An experimental sudy of cyclic deformation of extruded AZ61A magnesium alloy. International Journal of Plasticity 27, $768-787$.

Zhao, Z., Kuchnicki, S., Radovitzky, R., no, A. M. C., 2007. Influence of in-grain mesh resolution on the prediction of deformation textures in fcc polycrystals by crystal plasticity FEM. Acta Materialia 55, $2361-2373$. 